7th International Workshop on Astronomy and

Relativistic Astrophysics (IWARA 2016)

International Journal of Modern Physics: Conference Series

Vol. 45 (2017) 1760018 (4 pages)

(C) The Author(s)

DOI: $10.1142 / S 2010194517600187$

\title{
Quadrupole of a Composite Mass Optimization in Very High Speed Using FEM
}

\author{
Daniel Coppedé, Carlos Frajuca, Paulo R. M. Nogueira, Fabio S. Bortoli, \\ Marco A. Souza, Givanildo A. Santos, and Francisco Y. Nakamoto \\ Departamento de Mecânica \\ Instituto Federal de Educação, Ciência e Tecnologia de São Paulo (IFSP) \\ Rua Pedro Vicente, 625, 01109-010 São Paulo, Brazil \\ danielcoppede@gmail.com,frajuca@ifsp.edu.br,bortoli.fabio@gmail.com, \\ marcoantoniodesouza1@gmail.com,givanildo@ifsp.edu.br,nakamoto@ifsp.edu.br
}

Published 15 August 2017

\begin{abstract}
A experiment to measure the velocity of gravity is been planned. In order to achieve a reasonable signal in the detector, a very massive quadrupole mass is put to rotate at a very high rotation speed. In order to maximize the signal, the product mass and rotation radius squared much be as higher as possible. This work test a composite massquadrupole geometries in Finite Element Modeling (FEM) with the goal to maximize the signal
\end{abstract}

Keywords: Gravity Speed, Ultrahigh Speed, Control, Vacuum, Magnetic Bearing.

PACS numbers: 04.30.Db and 04.80.Cc

\section{Introduction}

The speed of gravity is one of the great mysteries of nature and one of the most difficult to be measured. Another way would be through the detection of gravitational waves but this is very difficult because until today only one where it was detected, but besides the detection of such wave a light counterpart must be found at the same time with different instruments, which makes this path almost impossible. It is best to issue a signal and detect it, which is intended in the continuation of this project.

In order to achieve this, it is proposed to develop a rotating system running at 600,000 rotations per minute (rpm) (theoretical limit is 2,000,000 rpm), this presents great challenge in the parts of magnetic suspension, direct drive, control of rotating systems and highly resistant materials.

This is an Open Access article published by World Scientific Publishing Company. It is distributed under the terms of the Creative Commons Attribution 4.0 (CC-BY) License. Further distribution of this work is permitted, provided the original work is properly cited. 


\section{Coppedé et al.}

\section{Theoretical Foundation}

The velocity of gravitational waves in the theory of general relativity equals the velocity of light in vacuum, $c$ (see Ref. ${ }^{1}$ ). Within the theory of special relativity, the constant $c$ is not exclusively about light; on the contrary, it is the highest speed possible for any interaction in nature. Formally, $\mathrm{c}$ is a conversion factor for changing the unit of time to the unit of space. ${ }^{2}$ This makes it the only speed that does not depend so much on the movement of an observer or a source of light and / or gravity.

The speed of physical changes in Isaac Newton's formulation of gravitational force law requires that every particle with mass respond instantaneously to any other particle with mass, regardless of the distance between them. In modern terms, Newtonian gravitation is described by the Poisson equation, whereby, when the mass distribution of a molting system, its gravitational field fits instantaneously. Therefore, the theory assumes that the speed of gravity is infinite. This hypothesis was adequate to explain all the phenomena with the accuracy of observation of the time. Until the 19th century only an anomaly in astronomical observations could not be reconciled with the Newtonian gravitational model of instantaneous action: the French astronomer Urbain Le Verrier determined in 1859 that the elliptical orbit of the planet Mercury precessed at a rate significantly different from that predicted by the Newtonian theory. ${ }^{3}$

The first attempt to combine a finite gravitational velocity with Newton's theory was made by Laplace in 1805. Based on Newton's law of force he considered a model in which the gravitational field is defined as a field of radiation or a fluid. Changes in the movement of the capture body are transmitted by some type of waves. ${ }^{4}$

In 1900 Hendrik Lorentz attempted to explain gravity based on his ether theory and Maxwell's equations. After proposing (and rejecting) a Le Sage type model, he assumed as Ottaviano Fabrizio Mossotti and Johann Karl Friedrich Zöllner that the attraction of opposite charged particles is stronger than the repulsion of equal charged particles. The resulting net force is exactly what is known as universal gravity, in which the speed of gravity is that of light. But Lorentz calculated that the value for Mercury's advance perihelion was very low. He wrote: "The special form of these terms may perhaps be modified. However, what has been said is sufficient to show that gravitation can be attributed to actions that are propagated with any greater velocity greater than that of light". 5

In 1908, Henri Poincaré examined Lorentz's gravitational theory and classified it as compatible with the principle of relativity, but (as Lorentz) criticized the imprecise indication of the advance of the perihelion of Mercury. ${ }^{6}$

The finite velocity of gravitational interaction in general relativity does not lead to types of problems such as the aberration of gravity that Newton was originally concerned because there is no such aberration in static field effects. Since the acceleration of the Earth relative to the Sun is small (that is, in a good approximation, the two bodies can be considered as traveling in straight lines over each other with a constant velocity) the orbital results calculated by general relativity are the Same as 
those of Newtonian gravity with instantaneous action at the same distance, because they are modeled by the behavior of a static field with constant velocity of relative motion, and without aberration for the forces involved. ${ }^{7}$

The first direct observation of gravitational waves, from the fusion of a pair of black holes, on September 14, 2015 (announced by the LIGO and Virgo collaborations on February 11, 2016 (see Ref. $\left.{ }^{8-10}\right)$ ) allowed a more direct measurement of its speed.

Another attempts are made to detect gravitational waves ${ }^{11-17}$.

\section{This Experiment}

Therefore, to directly measure the velocity of gravity, a tidal gravitational signal emitter and a detector are proposed to receive this signal. The speed will be obtained by the phase difference between the two devices weighted by distance. Since the speed of this signal must be very high, the device must rotate at a very high speed.

\section{Metodology}

Rotating Quadruple mass system can be seen on Fig. ??. On Fig. 1 the quadruple mass can be seen, it is made of carbon fiber (Ultimate Tensile Strength (UTS) equal

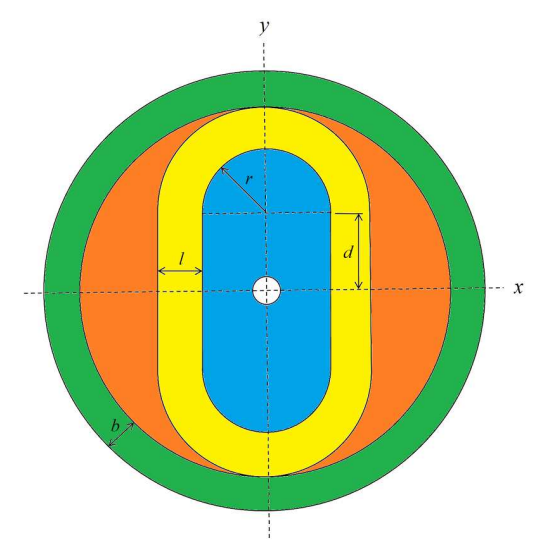

Fig. 1. The proposed model: Green and yellow: Carbon fiber, Blue: Maraging steel and Orange: Carbon fiber laminate

Table 1. Results of the quadruple mass dimensions optimization.

\begin{tabular}{|c|c|c|c|c|c|}
\hline $\begin{array}{c}\mathrm{d} \\
(\mathrm{cm})\end{array}$ & $\begin{array}{c}1 \\
(\mathrm{~cm})\end{array}$ & $\begin{array}{c}\mathrm{b} \\
(\mathrm{cm})\end{array}$ & $\begin{array}{c}\mathrm{r} \\
(\mathrm{cm})\end{array}$ & $\begin{array}{c}\mathrm{h} \\
(\mathrm{cm})\end{array}$ & $\begin{array}{c}\text { Mass } * \text { Ratio }^{2} \\
\left(k g * \mathrm{~cm}^{2}\right)\end{array}$ \\
\hline 4.85 & 3.2 & 0.4 & 4.23508 & 25.37015 & 206.96176 \\
\hline
\end{tabular}




\section{Coppedé et al.}

to $4137 \mathrm{MPa}$ and density equal to 1.75$)$, Maraging steel UTS equal to $2693 \mathrm{MPa}$ and density equal to 8.00) and carbon fiber laminate fiber (UTS equal to $1600 \mathrm{MPa}$ and density equal to 1.75). An optimization of the dimensions was made in order to achieve the bigger product mass times rotation radios squared, using the UTS as reference; the results are shown on Table 1.

\section{Conclusion}

The proposed model seems to have a reasonable product $M a s s *$ Radio $^{2}$ and, furthermore, produces a considerable gravitational signal to the experiment of measuring the velocity of gravity. The next step of this research is making a full simulation of the proposed model in FEM.

\section{References}

1. J. B. Hartle. Gravity: An Introduction to Einstein's General Relativity. (AddisonWesley, Boston, 2003) ISBN 981-02-2749-3, p. 332.

2. U. Le. Verrier, Lettre de M. Le Verrier à M. Faye Sur la Théorie de Mercure et Sur le Mouvement du Périhélie de Cette Planète, C. R. Acad. Sci. 49, 379 (1859).

3. P. S. Laplace, A Treatise in Celestial Mechanics, Vol. IV, Book X, Chapter VII (1805), translated by N. Bowditch (Chelsea, New York, 1966).

4. J. Zenneck, Gravitation, Encyklopädie der Mathematischen Wissenschaften mit Einschlussihrer Anwendungen (in German) 5, 25 (1903).

5. H. A. Lorentz. Considerations on Gravitation, Proc. Acad. Amsterdam 2, 559 (1900).

6. H. Poincaré, La dynamique de l'électron (PDF). Revue gnrale des sciences pures et appliques 19386 (1908). Reprinted in Poincar, Oeuvres Vol. IX, S. 551 and in Science and Method, (1908).

7. S. Carlip, Aberration and the Speed of Gravity, Phys. Lett. A 267, 23, 81 (2000). (arXiv:gr-qc/9909087)(1999).

8. LIGO Scientific Collab. and Virgo Collab. (B. P. Abbott et al.), Phys. Rev. Lett., 116 (6), 061102 (2016). Observation of Gravitational Waves from a Binary Black Hole Merger. arXiv:1602.03837(2016).

9. D. Castelvecchi and A. Witze, Einstein's Gravitational Waves Found at Last, Nature News doi:10.1038/nature.2016.19361. Retrieved 11 February 2016.

10. The Editorial Board (16 February 2016). The Chirp Heard Across the Universe. New York Times. Retrieved 16 February 2016.

11. C. Frajuca, K. L. Ribeiro, O. D. Aguiar, L. A. Andrade, P. J. Castro, N. S. Magalhães, and R. M. Marinho Jr. CQG 21, (2004).

12. C. Frajuca, K. L. Ribeiro, L. A. Andrade, W. F. Velloso Jr., J. L. Melo, O. D. Aguiar, and N. S. Magalhães, CQG 19, 1961 (2002).

13. W. W. Johnson and S. M. Merkowitz, Phys. Rev. Lett. 70, 2367 (1993).

14. W. W. Johnson and S. M. Merkowitz, Phys. Rev. D 56, 7513 (1997).

15. S. M. Magalhães, O. D. Aguiar, W. W. Johnson, and C. Frajuca. Gen. Relat. Grav. 29, 1511 (1997).

16. N. S. Magalhães, W. W. Johnson, C. Frajuca, and O. D. Aguiar. MNRAS 274, 670 (1995).

17. N. S. Magalhães, W. W. Jonhson, C. Frajuca, and O. D. Aguiar. Astrophysical Journal 475, 462 (1997). 